\title{
Potential of antibody-drug conjugates and novel therapeutics in breast cancer management
}

This article was published in the following Dove Press journal:

OncoTargets and Therapy

24 March 2014

Number of times this article has been viewed

\author{
Georgios D Lianos' \\ Konstantinos Vlachos ${ }^{2}$ \\ Odysseas Zoras ${ }^{3}$ \\ Christos Katsios' \\ William C Cho ${ }^{4}$ \\ Dimitrios H Roukos' \\ 'Centre for Biosystems and Genomic \\ Network Medicine, loannina \\ University, loannina, Greece; \\ ${ }^{2}$ Department of Surgery, loannina \\ University Hospital, loannina, Greece; \\ ${ }^{3}$ Department of Surgical Oncology, \\ Heraklion University Hospital, Crete, \\ Greece; ${ }^{4}$ Department of Clinical \\ Oncology, Queen Elizabeth Hospital, \\ Hong Kong
}

Correspondence: Dimitrios H Roukos

Centre for Biosystems and Genomic Network Medicine, loannina University, PO Box II 86, 45 II 0 loannina, Greece

Tel +302651007423

Fax+30265100 7094

Email droukos@uoi.gr

\begin{abstract}
Progress in the treatment of cancer over the past decade has been slow. Targeting a mutated gene of an individual patient tumor, tumor-guided agents, and the first draft of the human genome sequence have created an overenthusiasm to achieve personalized medicine. However, we now know that this effort is misleading. Extreme interpatient and intratumor heterogeneity, scarce knowledge in how genome-wide mutational landscape and epigenetic changes affect transcriptional processes, gene expression, signaling transduction networks and cell regulation, and clinical assessment of temporary efficacy of targeted drugs explain the limitations of these currently available agents. Trastuzumab and a few other monoclonal antibodies or small-molecule tyrosine kinase inhibitors (TKIs) represent an exception to this rule. By blocking ligand-binding receptor in patients with human epidermal growth factor receptor 2 (HER2) amplification and overexpression, trastuzumab added to chemotherapy in HER2-positive patients has been proven to provide significant overall survival benefit in both metastatic and adjuvant settings. Lapatinib, a small-molecule dual inhibitor (TKI) of both HER2 and EGFR (epidermal growth factor receptor) pathways, has an antitumor activity translated into progression-free survival benefit in HER2positive metastatic patients previously treated with a taxane, an anthracycline, and trastuzumab. Despite these advances, $\sim 25 \%$ of patients with HER2-positive breast cancer experience recurrence in the adjuvant setting, while in the metastatic setting, median survival time is 25 months. In this review, we discuss the safety, efficacy, and limitations of the trastuzumab emtansine (T-DM1) conjugate in the treatment of HER2-positive metastatic breast cancer. We also highlight Phase III randomized trials, currently underway, using either the T-DM1 conjugate or various combinations of monoclonal antibodies and TKIs. Moreover, in contrast with all these agents developed on the basis of "central dogma" of simplified reductionist transcription and single gene-phenotype linear relationship, we summarize the emerging, amazing era of next-generation, transcriptional circuitry and intracellular signaling network-based drugs guided by the latest advances in genome science and dynamics of network biology.
\end{abstract}

Keywords: trastuzumab emtansine, T-DM1, HER2 disease, targeted agents, cancer, genome, monoclonal antibodies

\section{Introduction}

Despite major advances in research, breast cancer still remains a big health problem. Worldwide, the incidence of the disease with $\sim 1,4000,000$ new cases each year, ${ }^{1}$ remains high, suggestive of slow progress in the prevention setting. In the treatment of women with established diagnosis, mortality rates have been improved, but the median survival time in the metastatic setting is only 25 months. ${ }^{2}$

With the standardization of systemic chemotherapy as the treatment of choice for most cancer types and the relatively modest expected improvement in both survival 
advantage and toxicity reduction, most interest and funding by the pharmaceutical industry and academia over the past decade have been given to targeted therapy. Targeting a single mutated gene and its encoded protein, targeted drugs can restore the deregulated signaling transduction pathway in which this mutated gene is involved. ${ }^{3}$ Considering that these agents are mostly active in cancer cells without affecting normal healthy cells, expectations were very high for both efficacy and low adverse effects.

Targeted therapy represents the major hope in the war against cancer and a substantial step towards personalized medicine. An overenthusiasm and explosion in tumor-guided drug development following the evidence of clinical success with trastuzumab (Herceptin ${ }^{\circledR}$; Genentech, Inc., San Francisco, CA, USA) in metastatic human epidermal growth factor receptor 2 (HER2)-positive breast cancer has been observed over the past decade. ${ }^{4}$

Indeed, more than 35 anticancer targeted drugs have been approved by the US Food and Drug Administration (FDA), and an additional $>150$ agents are in preclinical and clinical staging, aiming at the discovery of more effective therapies. In the vast majority, these anticancer agents target a single specific mutation or gene amplification. By inhibiting deregulated single-cellular signaling pathways, such agents can restore pathologic cell proliferation, survival, growth, apoptosis, invasion, angiogenesis, metabolism, and metastasis, which collectively are thought to be the hallmarks of cancer. ${ }^{5}$

\section{Slow progress in cancer treatment}

Despite the explosion in the single-gene-targeting approach, with intensive research efforts and major investment by the pharmaceutical industry and the public sector, the efficacy of these single signaling transduction pathway inhibitors is in most cases modest. Despite multiple enthusiastic publications on cancer therapy, current evidence-based medicine and science objectively show in top journal landmark reviews ${ }^{5-7}$ that the progress against cancer over the past decade is slow. This is translated into a few weeks or months survival prolongation in the metastatic setting, which is not surprising if we consider substantial limitations of currently available targeting therapies. ${ }^{5,7}$ The reasons for high intrinsic and acquired resistance rates to available targeting drugs include their temporary antitumor activity, ${ }^{5}$ lack of consideration of interpatient and intratumor heterogeneity, ${ }^{7-9}$ little attention to dynamics of transcriptional circuitry, and lack of a comprehensive view on how the cancer genome structure and molecular networks drive gene expression regulation. This understanding of extremely complex gene function is crucial for acquiring insights into how intracellular signaling networks in particular, and in general tumor microenvironment and cell-cell connectivity in tissues and organs, determine core cellular processes such as tumorigenesis and metastasis. $^{10-12}$

Despite this growing cancer complexity, now assessed by the latest clinical cancer genomics, ${ }^{5-12}$ substantial progress has been achieved for HER2-positive disease, which accounts for $20 \%$ of all breast cancer patients. Modern therapeutic decisions are based on an algorithm which considers traditional clinicopathologic criteria such as age, tumor stage, nodal status, histological grade, and estrogen receptors (ER) and HER2 status. Although HER2-positive patients exhibit higher risk for recurrence or disease progression than the HER2-negative subset of patients for which no targeted therapy has become available, the discovery of anti-HER2 agents can alter this negative prognosis. Indeed, the identification of the HER2 pathway and its dysfunction when the HER 2 gene is amplified, resulting in HER2 overexpressing breast cancer cells, has led to the development of the anti-HER2 monoclonal antibody (mAb) trastuzumab. Since 2001 and 2005, when Phase III randomized trials showed that trastuzumab addition to chemotherapy in HER2-positive breast cancer significantly prolongs overall survival (OS) in metastatic $^{4}$ and adjuvant ${ }^{13,14}$ settings, trastuzumab has been the standard first-line therapy for these patients. Despite these significant advances, recurrence and disease progression rates still remain alarmingly high. The single agent trastuzumab emtansine (T-DM1) provides a potentially improved clinical outcome. In this review, we discuss the safety and efficacy of T-DM1 conjugate as well as its limitation in the treatment of metastatic HER2-positive breast cancer. In addition, we summarize Phase III trials currently underway, comparing both this single agent with various regimens and a variety of new combinations of mAbs with TKIs. Finally, we highlight novel future directions in discovering new druggable agents. Innovative approaches are being developed by integrating breakthrough technologies. New methodological sophisticated strategies in the post-ENCODE era are summarized aiming to the discovery of next-generation biomarkers and drugs able to disrupt dynamic cancer molecular circuitry.

\section{Antibody-drug conjugates}

Cytotoxic chemotherapy has long been considered as standard systemic treatment in cancer, with substantial response rates and clinical benefits, but it is associated with relatively high adverse events. Methods to improve both the selectivity and the therapeutic index of cytotoxic drugs have been developed. 
One of the top areas of interest is targeted drug carriers such as antibodies; currently, this is the main role of antibodydrug conjugates (ADCs). ${ }^{15,16}$ Interestingly, cytotoxic drugs are believed to be attached to ADCs via chemical linkers to antibodies that recognize cancer cell antigens; the most important point is that the cytotoxic drug is delivered only to the tumor cells. For this purpose, ADCs must be considered as "ideal" delivery systems for antitumor cytotoxic drugs. ${ }^{15,17}$ Critical areas for ADC development include target antigen selection and stability of the linker between the complex (drug-antibody) as well. Other crucial parameters include drug-to-antibody ratio and the effects of drug conjugation on antibody potential.

Up to 2 years ago, only two ADCs had gained the necessary approval by the US FDA. ${ }^{18}$ Interestingly, the ADC gemtuzumab-ozogamicin was approved in 2000 for the management of relapsed CD33-positive acute myeloid leukemia. Recently, brentuximab-vedotin was approved for patients with Hodgkin lymphoma after failure of stem cell transplant or after failure of two prior multi-agent chemotherapy regimens. ${ }^{19}$ Gemtuzumab-ozogamicin was withdrawn from clinical use. Studies after its approval by the US FDA showed no benefit of adding this ADC to chemotherapy for the treatment of patients with acute myeloid leukemia. ${ }^{20}$

As breast cancer is, and will remain in the future, a worldwide major health problem for the female population, ${ }^{21}$ ongoing research in the ADC field has developed methods and techniques for the design of more effective therapeutics.

The HER2 receptor, one of four receptors (epidermal growth factor receptor [EGFR] or HER1, HER2, HER3, and HER4) of the EGFR family, is a transmembrane receptor protein which is constituted from an extracellular and an intracellular domain. The EGFR or ErbB family of receptor tyrosine kinases has a crucial role in tumorigenesis. Various therapeutics targeting these receptors have been studied and approved for the management of several types of cancer. Recent data have shown that the administration of two inhibitors, against for example EGFR or ErbB2 family members, acts synergically, increasing significantly the antitumor activity as compared with the administration of single agents. For example, combined treatment with trastuzumab and lapatinib, a dual inhibitor of EGFR and HER2, leads to a complete regression of BT474 breast cancer xenografts, which have an amplified $E R B B 2$ gene; on the other hand, researchers have shown that single-agent treatment causes only partial tumor regression. These studies were also extended to an MCF7 breast cancer xenograft model expressing transfected HER2. ${ }^{22}$
The ADC strategy can be particularly effective in HER2positive breast cancer ${ }^{23}$ considering the substantial clinical response to chemotherapy plus trastuzumab in patients with HER2-overexpressing breast cancer. ${ }^{24-26}$ Before we address the ado-T-DM1 approach, we provide here a description of trastuzumab treatment.

\section{Trastuzumab}

By blocking the ligand-binding extracellular "part" of the HER2 receptor, the $\mathrm{mAb}$ trastuzumab inhibits the pathologic downstream signal transduction of the HER2 pathway in HER2-overexpressing breast cancer. In the adjuvant setting, early Phase III trials with short 1- or 2-year follow-up have shown that this antibody added to chemotherapy reduces the relative risk of recurrence by $50 \%$ and significantly increases OS rate..$^{27,28}$ Analysis of all related studies confirms that anti-HER2 treatment with trastuzumab is effective only in HER2-positive disease. ${ }^{29}$

More recently, the Herceptin Adjuvant Trial (HERA) ${ }^{30}$ has released longer follow-up results of a Phase III, trastuzumab-based study. At a median of 8 years follow-up, there were 471 events of disease-free survival (DFS) reported in 1,552 patients $(30.3 \%)$ in the 1-year trastuzumab treatment group and 570 events in 1,697 patients in the observation (control) group (33.6\%). Moreover, 278 deaths were observed in the 1-year group (17.9\%) versus 350 deaths observed in the standard group (20.6\%). The researchers concluded that all the results favored the 1-year trastuzumab treatment group.

What is the main message from this 8-year follow-up study, taking into account the general rule of temporary antitumor activity of nearly all cancer-targeted drugs available? First, the differences in DFS and OS remain significant at 8 years. Therefore, in contrast to most other tumor-guided agents, trastuzumab is able to provide a durable survival benefit. Second, despite this long-term statistically significant antitumor activity, the clinical response rate and absolute survival benefit is small (3.3\% DFS). This finding suggests the need for developing more effective drugs with T-DM1 conjugate to represent a potential alternative.

What is the optimal duration of treatment with trastuzumab? It is still unclear whether 6 months, 1 year, or 2 years provide the higher benefit. In the same international, multicenter, randomized, open-label, Phase III trial (HERA), ${ }^{30}$ the researchers compared treatment with trastuzumab for 1 and 2 years after standard neoadjuvant chemotherapy, adjuvant chemotherapy, or both. ${ }^{30}$ The primary endpoint of this well designed trial was DFS. The comparison of 2 years versus 1 year of trastuzumab 
treatment involved 3,105 patients. In the 1-year group, 367 events of DFS in 1,552 patients $(23.6 \%)$ were reported, and in the 2-year group, 367 events in 1,553 patients (hazard ratio [HR] 0.99, 95\% confidence interval [CI] 0.85-1.14, $P=0.86$ ). Interestingly, 2 years of adjuvant trastuzumab is no more effective than 1 year of treatment for patients with HER2-positive early breast cancer. Moreover, 1 year of treatment provides a significant DFS and OS benefit compared with the observation group and remains the standard of care. In conclusion, the optimum duration of adjuvant trastuzumab remains unknown, but it is now widely accepted that it is likely to be 12 months or less. The next step is to evaluate and analyze studies with treatment durations shorter than 12 months. ${ }^{30-33}$

\section{Resistance to trastuzumab}

Despite the trastuzumab-based survival benefit for HER2positive early, advanced, and metastatic breast cancer, the major problem today is how to overcome the intrinsic and acquired resistance. ${ }^{28}$ Indeed, this tumor nonresponsiveness to chemotherapy plus trastuzumab is reflected by a $70 \%$ DFS rate at 8 years in the adjuvant setting and is near $25 \%$ median OS in the metastatic setting. Exciting research has focused on understanding molecular mechanisms underlying this resistance, aiming to develop novel, more effective drugs with higher clinical response rates. In this way, lapatinib, which is a dual inhibitor of HER2 and EGFR, is used in combination with capecitabine. This therapeutic approach has shown antitumor activity and prolongation of the time to disease progression in patients who have previously been treated with trastuzumab, an anthracycline, and a taxane. ${ }^{34}$ This drug combination was, until recently, the standard alternative to trastuzumab treatment for relapsed or refractory HER2-positive breast cancer.

In summary, the discovery of the anti-HER2 agent trastuzumab gained regulatory approval, not only for the metastatic but also for the adjuvant setting, improving OS, with durable effect in patients with HER2-positive breast cancer. In contrast to most other targeted drugs, trastuzumab represents a triumph of translational medical research. Today trastuzumab still remains an isolated success for adjuvant setting. No other targeted drug for major solid cancers is used in this early stage of disease. ${ }^{28}$ However, the absolute survival benefit is small because resistance and relapse or disease progression rates with fatal outcome still remain high.

\section{Trastuzumab emtansine (T-DMI)}

The single agent T-DM1 is an ADC that incorporates the HER2-targeted antitumor capacities of trastuzumab with the cytotoxic properties of the microtubule-inhibitory agent DM1 (derivative of maytansine), ${ }^{35,36}$ the antibody and the cytotoxic agent are described to be conjugated by a stable linker. Interestingly, T-DM1 allows intracellular drug delivery specifically to HER2-overexpressing cells, thereby improving the therapeutic rate and minimizing the exposure of normal tissue cells to the complex. It seems that T-DM1 is internalized upon binding to HER2-overexpressing tumor cells. Moreover, it is the first HER2-targeted ADC with a stable and unique linker. ${ }^{37,38}$ T-DM1 is an ADC that is currently being investigated in various clinical trials. Early in its development, studies were conducted to identify the optimal linker to conjugate trastuzumab to DM1. Interestingly, in preclinical studies, it was shown that linking DM1 to trastuzumab via a non-reducible thioether yielded superior activity, improved pharmacokinetics, and presented less toxicity compared with trastuzumab linked to a maytansinoid via a disulfide linker. Furthermore, the T-DM1 was shown to be selective for HER2-positive cells, displayed enhanced potency compared with trastuzumab alone in vitro, and retained activity against trastuzumab-resistant cells in vitro and in vivo. ${ }^{39}$ In other words, T-DM1 is an agent that combines an antibody and a cytotoxic agent, which are conjugated by means of a stable linker. Because the intracellular delivery specifically to breast cancer cells overexpressing HER2 is feasible for the T-DM1 drug, it maximizes the efficacy of this drug and at the same time minimizes the exposure of the drug in normal tissue, decreasing adverse effects.

The near future will determine the exact role of T-DM1 in the current therapeutic "armamentarium" for HER2metastatic breast cancer. ${ }^{40,41}$

\section{Clinical studies}

On the basis of several positive Phase II clinical trials, a Phase III, randomized trial, has been completed. The results of all these completed and published studies are summarized in Table 1.

\section{Phase II studies}

Three well conducted Phase II single-arm studies of T-DM1 have been published ${ }^{42-44}$ (Table 1). Interestingly, in a trial of 112 patients who had received chemotherapy and had tumor progression after HER2-targeted therapy, T-DM1 was correlated with an objective response rate of $25.9 \%$ (95\% CI: $18.4 \%-34.4 \%){ }^{42}$ The median duration of response reported was about 9.5 months. In addition, the median progression-free survival (PFS) was 4.6 months (95\% CI: 3.9-8.6 months). Moreover, T-DM1 was well tolerated by 
Table I Completed Phase II and Phase III trial results for T-DMI

\begin{tabular}{|c|c|c|c|c|}
\hline Study/phase & Number of patients & Arms & Endpoints & HR; 95\% Cl \\
\hline EMILIA Trial ${ }^{45}$ & $991 *$ & T-DMI vs lapatinib and capecitabine & MOS: 30.9 vs & HR 0.68; $95 \% \mathrm{Cl} 0.55-0.85$ \\
\hline Phase III & & & 25.1 months & $P<0.001$ \\
\hline Burris et $\mathrm{al}^{42}$ & 112 & Single-arm study; patients pretreated & ORR: $25.9 \%$ & $95 \% \mathrm{Cl} 18.4 \%-34.4 \%$ \\
\hline Phase II & & with chemotherapy & PFS: 4.6 months & $95 \% \mathrm{Cl} 3.9-8.6$ months \\
\hline Krop et $\mathrm{al}^{43}$ & 110 & Single-arm study; patients pretreated & ORR: $32.7 \%$ & $95 \% \mathrm{Cl} 24.1-42.1 \%$ \\
\hline Phase II & & $\begin{array}{l}\text { with anthracycline, trastuzumab, taxane, } \\
\text { capecitabine, and lapatinib therapy }\end{array}$ & PFS: 7.3 months & \\
\hline Hurvitz et $\mathrm{al}^{44}$ & $137 * *$ & T-DMI vs trastuzumab plus docetaxel & ORR: $64 \%$ vs $58 \%$ & HR 0.594, \\
\hline $\begin{array}{l}\text { Phase II } \\
\text { randomized }\end{array}$ & & & PFS: I 4.2 vs 9.2 months & $P=0.035$ \\
\hline
\end{tabular}

the patients enrolled in the study. The most adverse events were of grade 1-2, while the most common grade 3 adverse events were hypokalemia, thrombocytopenia, and fatigue. ${ }^{42}$ Another interesting point was that no dose-limiting cardiotoxicity was observed. ${ }^{42}$

Furthermore, in a study of 110 patients with HER2overexpressing metastatic breast cancer who had received previous anthracycline, trastuzumab, taxane, capecitabine, and lapatinib therapy, with evidence of progressive disease on their last regimen, T-DM1 monotherapy led to an objective response rate of $32.7 \%$ ( $95 \%$ CI: $24.1 \%-42.1 \%$ ), a median duration of response of 7.2 months, and a median PFS of 7.3 months. T-DM1 was well tolerated in this pretreated population, with no cardiac events requiring dose reductions or additional new safety concerns. ${ }^{43}$ The final results from a randomized Phase II trial of T-DM1 versus trastuzumab plus docetaxel in HER2-positive metastatic breast cancer involved 137 patients and were recently published. ${ }^{44}$ In these patients, with a median follow-up of about 14 months, an important difference in the PFS was seen in patients receiving T-DM1 (14.2 versus 9.2 months) (HR 0.594, $P=0.035$ ). In this randomized Phase II study, first-line treatment with
T-DM1 for patients with HER2-positive metastatic breast cancer provided a significant improvement in PFS, associated with a notable safety profile.

\section{Phase III randomized trials}

On the basis of these promising results, a Phase III randomized trial has been completed (Table 1) and another three Phase III studies are underway (Table 2). The EMILIA trial, ${ }^{45}$ which is a randomized, multicenter, Phase III, openlabel study, evaluated the safety and efficacy of T-DM1 compared with lapatinib plus capecitabine in patients with HER2-positive advanced or metastatic breast cancer. All patients enrolled had a prior treatment with trastuzumab and taxane-based chemotherapy. From February 2009 through October 2011, a total of almost 1,000 patients were enrolled at 213 centers. It is reported that 496 patients were randomly assigned to receive lapatinib plus capecitabine and 495 to receive T-DM1. The primary and secondary endpoints were median PFS, median OS, and objective response rate along with adverse event rates. Verma et $\mathrm{a}^{45}$ reported that median PFS was 9.6 months in the T-DM1 group versus 6.4 months in the lapatinib plus capecitabine group (HR for progression

Table 2 Phase III studies with T-DMI currently underway

\begin{tabular}{llll}
\hline Study & Numberof patients & Arms & Median overall survival (months) \\
\hline MARIANNE Trial ${ }^{46}$ & $1,092^{* *}$ & T-DMI with or without pertuzumab & Underway \\
& & vs trastuzumab and taxane & Underway \\
TH3RESA Trial ${ }^{47}$ & $795^{* * *}$ & T-DMI vs physician's choice & Underway \\
KATHERINE Trial & $1,484^{* * * *}$ & T-DMI vs trastuzumab for women & with residual tumor \\
\hline
\end{tabular}

Notes: **Patients with HER2-positive progressive or recurrent locally advanced or metastatic breast cancer previously untreated (first-line treatment in metastatic setting), or patients who may have had chemotherapy and trastuzumab or lapatinib in adjuvant setting; ****atients with metastatic or unresectable locally advanced or recurrent HER2-positive breast cancer who have received at least two prior regimens of HER2-directed therapy; **** patients with HER2-positive primary breast cancer who have residual tumor present pathologically in the breast or axillary lymph nodes following preoperative therapy.

Abbreviations: HER2, human epidermal growth factor receptor 2; T-DMI, trastuzumab emtansine; vs, versus. 
or death from any cause, $0.65 ; 95 \%$ CI, 0.55-0.77; $P<0.001)$. Moreover, the median OS was 30.9 months versus 25.1 months; (HR for death from any cause, 0.68 ; 95\% CI, $0.55-0.85 ; P<0.001)$. Furthermore, the objective response rate was higher in patients treated with T-DM1 $(43.6 \%$ versus $30.8 \%$ with lapatinib plus capecitabine; $P<0.001$ ). The researchers concluded that all the results favored the T-DM1 group. Of note, the rates of adverse events of grade 3 or above were higher with lapatinib plus capecitabine than with T-DM1 (57\% versus 41\%), while the rates of thrombocytopenia and increased serum aminotransferase levels were higher with T-DM1. On the other hand, the incidences of diarrhea, nausea, and palmar-plantar erythrodysesthesia were higher with lapatinib plus capecitabine. The OS seemed to be improved for patients receiving T-DM1, and the incidence of cardiac toxicity was not increased. There was one death due to toxicity in the T-DM1 group and five deaths in the capecitabine plus lapatinib group.

Analyzing all these data, we can conclude that the EMILIA study is a positive Phase III trial meeting all the primary and secondary endpoints, providing strong evidence for the efficacy and safety of T-DM1. ${ }^{28}$ In this Phase III study, the most positive finding was that this single agent extended life by 6 months. This is a crucial achievement considering that most available molecularly targeted drugs added to chemotherapy in the treatment of various cancer types provide only a PFS benefit but no OS prolongation in the metastatic setting. This survival benefit was observed regardless of the line of therapy in patients with metastatic disease and was seen in patients with a disease-free interval of less than 6 months after completion of trastuzumab-based therapy in the adjuvant or neoadjuvant setting. In addition to OS benefit, this single-agent treatment was associated with a lower side-effects profile. However, objective evaluation and comparison of OS curves in the EMILIA study proves that the significant durable effect of T-DM1 is limited to 6 months benefit as compared with the lapatinib capecitabine group, and the survival curves become identical at 32 months after treatment. This finding underlines the development of resistance and loss of antitumor activity and clinical response beyond a 6-month therapeutic gain as compared with capecitabine plus lapatinib treatment in metastatic HER2-positive breast cancer.

In summary, T-DM1 provides high therapeutic potential, and can be recommended as a new standard second-line treatment in relapsed or refractory HER2-positive breast cancer after chemotherapy with trastzuzumab or lapatinib treatment. However, even with T-DM1, median OS is $\sim 31$ months with current and emerging research to explore new drug combinations and innovative approaches with the hope to further improve clinical response and survival.

\section{Future perspectives}

With the clinical benefit of T-DM1 established, several new therapeutic options including either this single agent or other combinations of mAbs and TKIs are being explored.

Table 2 summarizes the T-DM1 Phase III trials currently underway. Interestingly, the MARIANNE study, which is a randomized, three-arm, multicenter, Phase III study to evaluate the efficacy and safety of T-DM1 combined with pertuzumab or T-DM1 combined with pertuzumab placebo (blinded for pertuzumab), versus the combination of trastuzumab plus taxane, as first-line treatment in HER2-positive progressive or recurrent locally advanced or metastatic breast cancer is comparing the efficacy and safety of single-agent T-DM1 versus T-DM1 plus pertuzumab versus trastuzumab plus a taxane for the first-line treatment of HER2-positive, metastatic, or locally recurrent breast cancer. A total of 1,092 patients have been enrolled. The primary endpoint is PFS, while the secondary endpoints include safety, overall response rate, OS, duration of response, and quality of life..$^{39,46}$

The T-DM1 in comparison with treatment of physician's choice in patients with HER2-positive breast cancer who have received at least two prior regimens of HER2-directed therapy (TH3RESA study) is comparing third-line T-DM1 to physician's choice of treatment in HER2-positive metastatic breast cancer. This randomized, two-arm study, involving 792 patients, is evaluating the efficacy and safety of T-DM1 compared with treatment of the physician's choice in patients with metastatic or unresectable locally advanced or recurrent HER2-positive breast cancer. The duration of study treatment is until disease progression or unacceptable toxicity occurs. The primary outcomes are objective response rate by independent review and OS. Secondary outcomes include PFS, the duration of objective responses, safety, and clinical benefit rate, defined as the proportion of patients achieving an objective response or stable disease for at least 6 months from randomization. ${ }^{39,47}$

Interestingly, the Phase III KATHERINE study of T-DM1 versus trastuzumab as adjuvant therapy for patients with HER2-positive primary breast cancer who have residual tumor present pathologically in the breast or axillary lymph nodes following preoperative therapy was initiated some months ago. The estimated enrollment is 1,484 patients, and the first results are expected with great interest. ${ }^{39,48}$ There is 
no doubt that T-DM1 may set a new standard for anticancer therapy as a drug with minimal toxicity and significant efficacy in previously treated HER2-positive breast cancer patients.

Moreover, in the top of the scientific interest is the combination of two or more antibodies and TKIs that act synergically. Table 3 summarizes the combinations of two or more mAbs and TKIs in Phase III trials. The researchers believe that pertuzumab binds an epitope on ERBB2 that does not overlap with the binding epitope of trastuzumab and for this reason has a complementary action to that of trastuzumab. This was evaluated in a Phase II trial where the combination of trastuzumab and pertuzumab was well tolerated and showed promising results, with an overall response rate of $24.2 \%$ and a median PFS of 5.5 months. ${ }^{49}$ In the light of these encouraging results, the CLEOPATRA study was conducted. This trial examined the activity of combined trastuzumab and docetaxel chemotherapy with or without pertuzumab in a Phase III randomized study in patients with HER2-positive metastatic breast cancer. The median PFS was 18.5 months in the pertuzumab group and 12.4 months in the control group (HR 0.62, 95\% CI 0.51-0.75; P<0.001). Moreover, the OS rates are in favor of the trastuzumab-pertuzumab group of patients. ${ }^{50}$ Based on this important trial, the US FDA approved, 1 year ago, pertuzumab (PERJETA ${ }^{\mathrm{TM}}$; Genentech) in combination with trastuzumab and docetaxel for the treatment of HER2-positive metastatic breast cancer. ${ }^{51}$

Moreover, the combination of an antibody with a TKI showed promising results in various types of cancers and can possibly represent an important therapeutic tool for the near future. Interestingly, in the breast cancer setting, a Phase III study of lapatinib-TKI and trastuzumab showed superior PFS and OS rates compared with lapatinib in patients with advanced ERBB2-positive cancer who had progressed after having previously been treated with trastuzumab (HR 0.73 , 95\% CI 0.57-0.93; $P=0.008) .{ }^{52}$ Similar results were obtained in the neoadjuvant setting. Pathological complete response was significantly higher in the combination group who were given lapatinib and trastuzumab (51.3\%; 95\% CI 43.1-59.5) (Table 3). ${ }^{53}$ Dual therapy with trastuzumab and lapatinib, however, shows important benefit for HER2-positive breast cancer. $^{51-53}$

\section{Beyond current-day medical research}

A future goal with highly effective drug combinations on the basis of personal mutational landscape and transcriptome architecture is the next-generation genome diagnostics-based therapeutics.

Breakthrough sequencing technologies ${ }^{54}$ now reveal the importance not only of protein-coding sequences for identifying intragenetic variation but also noncoding, regulatory natural variants which affect transcription and gene expression, ensuring biodiversity in human physiology and evolution. ${ }^{55}$ Genome-wide association studies have shown that most $(88 \%)$ of disease-associated variants in susceptibility loci are within the noncoding region of the human genome. ${ }^{56}$ Therefore, protein-coding and noncoding mapping of the genome using next-generation sequencing technologies for whole-genome sequencing/whole-exome sequencing and RNA (ribonucleic acid)-sequencing can improve our understanding of cancer and other common disease pathogenesis. ${ }^{57,58}$ Understanding inter-individual genetic variation as a cause of diversity in phenotype ${ }^{55}$ and interpatient tumor genetic and genomic mutational heterogeneity, ${ }^{7,8}$ or both coding and noncoding DNA (deoxyribonucleic acid)

Table 3 Combinations of two or more mAbs and TKIs in Phase III trials

\begin{tabular}{|c|c|c|c|c|}
\hline Study & $\begin{array}{l}\text { Number of } \\
\text { patients }\end{array}$ & Arms & Endpoints & HR; 95\% Cl \\
\hline $\begin{array}{l}\text { CLEOPATRA }^{50} \text { (combination } \\
\text { of } \mathrm{mAbs} \text { ) }\end{array}$ & 808 & $\begin{array}{l}\text { Placebo }+ \text { trastuzumab }+ \text { docetaxel } \\
\text { (control group) vs pertuzumab }+ \\
\text { trastuzumab }+ \text { docetaxel } \\
\text { (pertuzumab group) }\end{array}$ & PFS: 12.4 vs 18.5 months & $\begin{array}{l}\text { HR 0.62; } \\
95 \% \mathrm{Cl} 0.5 \mathrm{I}-0.75 \\
P<0.00 \mathrm{I}\end{array}$ \\
\hline $\begin{array}{l}\text { Blackwell et } \mathrm{l}^{52} \text { (combination } \\
\text { of } \mathrm{mAbs} \text { and } \mathrm{TK} / \mathrm{s} \text { ) }\end{array}$ & $296 *$ & Lapatinib vs lapatinib + trastuzumab & PFS & $\begin{array}{l}\text { HR } 0.73 ; \\
95 \% \text { Cl 0.57-0.93; } \\
P=0.008\end{array}$ \\
\hline $\begin{array}{l}\text { Baselga et } \mathrm{al}^{53} \text { (combination } \\
\text { of } \mathrm{mAbs} \text { and } \mathrm{TKI} \text { s in } \\
\text { neoadjuvant setting) }\end{array}$ & $455^{* *}$ & $\begin{array}{l}\text { Lapatinib vs trastuzumab vs lapatinib }+ \\
\text { trastuzumab }\end{array}$ & $\begin{array}{l}\text { PCR was significantly higher in } \\
\text { the combination group given } \\
\text { lapatinib and trastuzumab than } \\
\text { in monotherapy groups }\end{array}$ & $\begin{array}{l}51.3 \% \\
95 \% \mathrm{Cl} 43.1-59.5\end{array}$ \\
\hline
\end{tabular}

Notes: *Patients with HER2-positive metastatic breast cancer who presented progression on previous trastuzumab regimens; **patients with HER2-positive early breast cancer with tumours $>2 \mathrm{~cm}$ in diameter.

Abbreviations: $\mathrm{Cl}$, confidence interval; HR, hazard ratio; mAbs monoclonal antibodies; PCR, pathological complete response; PFS, progression-free survival; TKI, tyrosine kinase inhibitor; vs, versus. 
and RNA is crucial for understanding, preventing, and treating cancer.

However, the current high-throughput sequencing-based confirmation of the previous concept of clonal mutation evolution, interpatient, and perhaps, intra-tumor heterogeneity ${ }^{7,8}$ dramatically increases the challenge in how to predict tumor responsiveness and select the best possible therapeutic agent combination treatment for an individual patient. However, this is not the only problem in the effort to reach robust personalized treatment. Beyond a patient's mutational landscape assessment, the next and bigger challenge is to understand how this heterogeneous genomic aberration affects individual genome function, cellular signaling networks, and gene expression regulation. ${ }^{59}$ There is now a clear consensus that personalized clinical medicine is much more complex than it was first thought. Understanding transcriptional circuitry, ${ }^{60,61}$ driving gene expression regulation, represents a daunting challenge which requires innovative developments in both technologies and methods.

New exciting research efforts are emerging in exploring genome-wide molecular mechanisms regulating gene expression and are behind cancer cell progression and metastasis. Now, in the post-ENCODE era, ${ }^{62}$ breakthrough technologies including high-throughput sequencing and arrays, as well as livingcell imaging techniques, including visualization of interacting molecules using biosensors coupled with novel computational and mathematical approaches, allow the study of molecular interactions, transcriptional circuitry, and gene expression regulation through intracellular signaling networks. ${ }^{63-65}$ As we are now moving away from the standard, linear approach of simplified single-gene transcription-dominated human biology and medicine, which lasted $\sim 60$ years, to a much more complex transcriptional network driving gene regulation, ${ }^{66}$ the new postENCODE age of genome network medicine (GNM) is just now beginning. ${ }^{67}$ The objective of GNM is patient-derived sampling analysis for biospecimen-based cancer genome architecture in large-scale international genomic studies. ${ }^{68,69}$ It is expected that these patient-derived genomics will not only complete the genetic mutations catalogue for each cancer type but also can provide revolutionary information on how mutational and epigenetics genome-wide landscape affects transcriptional regulatory networks, gene function and cancer cell metastatic or non-metastatic capacity. Although this goal appears overambitious, it is perhaps the single way to dramatically improve cancer patient outcome. This rational network-based approach, combining advances in genome science and network biology, provides high hopes for the future development of robust biomarkers for assessing sensitivity or resistance to available agents and the discovery of next-generation, network-based drugs. The era of GNM has begun. ${ }^{67}$

\section{Conclusion}

The single-agent T-DM1 after a recent regulatory approval has been a standard treatment for trastuzumab plus chemotherapy refractory or relapsed HER2-positive breast cancer. ${ }^{39,68,69}$ Currently underway Phase III trials can expand therapeutic indications including T-DM1 as first-line therapy in the metastatic and adjuvant settings. Other combinations of mAbs, or mAbs and TKIs, have been completed or are underway evaluating potential more effective therapeutics in HER2-positive breast cancer. However, all these drugs have been developed on the basis of "central dogma" of simplified transcription. In contrast to this one gene-phenotype linear relationship, a new post-ENCODE era has begun for developing transcriptional networks and biological systems interaction-based next-generation drugs. Rapid innovative developments in genome science and network biology, using breakthrough mapping and visualizing technologies and complex bioinformatics for analytical approaches of big data along with new and dynamic computational strategies shape a new research environment in academia and the pharmaceutical industry. The expectation for the future or clinical GNM is adapted and transformed into a real-world, patient-derived approach.

\section{Disclosure}

The authors declare no conflicts of interest in this work.

\section{References}

1. Malvezzi M, Bertuccio P, Levi F, et al. European cancer mortality predictions for the year 2013. Ann Oncol. 2013;24(3):792-800.

2. Siegel R, Naishadham D, Jemal A. Cancer statistics, 2013. CA Cancer J Clin. 2013;63(1):11-30.

3. Rask-Andersen M, Almén MS, Schiöth HB. Trends in the exploitation of novel drug targets. Nat Rev Drug Discov. 2011;10(8):579-590.

4. Slamon DJ, Leyland-Jones B, Shak S, et al. Use of chemotherapy plus a monoclonal antibody against HER2 for metastatic breast cancer that overexpresses HER2. $N$ Engl J Med. 2001;344(11):783-792.

5. Hanahan D, Weinberg RA. Hallmarks of cancer: the next generation. Cell. 2011;144(5):646-674.

6. Vogelstein B, Papadopoulos N, Velculescu VE, Zhou S, Diaz LA Jr, Kinzler KW. Cancer genome landscapes. Science. 2013;339(6127): 1546-1558.

7. Klein CA. Selection and adaptation during metastatic cancer progression. Nature. 2013;501(7467):365-372.

8. Bedard PL, Hansen AR, Ratain MJ, Siu LL. Tumour heterogeneity in the clinic. Nature. 2013;501(7467):355-364.

9. Roukos DH. Beyond HER2 and trastuzumab: heterogeneity, systems biology, and cancer origin research may guide the future for personalized treatment of very early but aggressive breast cancer. J Clin Oncol. 2010;28(17):e279-e280.

10. Cancer Genome Atlas Network. Comprehensive molecular portraits of human breast tumors. Nature. 2012;490(7418):61-70. 
11. Stamatoyannopoulos JA. What does our genome encode? Genome Res. 2012;22(9):1602-1611.

12. Roukos DH, Baltogiannis GG, Katsouras CS, et al. Novel nextgeneration sequencing and networks-based therapeutic targets: realistic more effective drug design and discovery. Curr Pharm Des. 2014;20(1): $11-22$.

13. Piccart-Gebhart MJ, Procter M, Leyland-Jones B, et al. Trastuzumab after adjuvant chemotherapy in HER2-positive breast cancer. $N$ Engl JMed. 2005;353(16):1659-1672.

14. Romond EH, Perez EA, Bryant J, et al. Trastuzumab plus adjuvant chemotherapy for operable HER2-positive breast cancer. $N$ Engl J Med. 2005;353(16):1673-1684.

15. Barginear MF, John V, Budman DR. Trastuzumab-DM1: a clinical update of the novel antibody-drug conjugate for HER2-overexpressing breast cancer. Mol Med. 2012;18:1473-1479.

16. Ducry L, Stump B. Antibody-drug conjugates: linking cytotoxic payloads to monoclonal antibodies. Bioconjug Chem. 2010;21:5-13.

17. Senter PD. Potent antibody drug conjugates for cancer therapy. Curr Opin Chem Biol. 2009;13:235-244.

18. Erickson HK, Lewis Phillips GD, Leipold DD, et al. The effect of different linkers on target cell catabolism and pharmacokinetics/ pharmacodynamics of trastuzumab-maytansinoid conjugates. Mol Cancer Ther. 2012;11(5):1133-1142.

19. Pro B, Advani R, Brice P, et al. Brentuximab vedotin (SGN-35) in patients with relapsed or refractory systemic anaplastic large-cell lymphoma: results of a Phase II study. J Clin Oncol. 2012;30:2190-2196.

20. US Food and Drug Administration (FDA) [homepage on the Internet]. Mylotarg (gemtuzumab ozogamicin): market withdrawal. Silver Spring, MD: FDA; 2010 [updated June 21, 2010; cited January 11, 2013]. Available from: http://www.fda.gov/safety/ medwatch/safetyinformation/safetyalertsforhumanmedicalproducts/ ucm216458.htm. Accessed January 27, 2014.

21. Jemal A, Bray F, Center MM, Ferlay J, Ward E, Forman D. Global cancer statistics. CA Cancer J Clin. 2011;61:69-90.

22. Rimawi MF, Wiechmann LS, Wang YC, et al. Reduced dose and intermittent treatment with lapatinib and trastuzumab for potent blockade of the HER pathway in HER2/neu-overexpressing breast tumor xenografts. Clin Cancer Res. 2011;17:1351-1361.

23. Hurvitz SA, Kakkar R. The potential for trastuzumab emtansine in human epidermal growth factor receptor 2 positive metastatic breast cancer: latest evidence and ongoing studies. Ther Adv Med Oncol. 2012;4(5):235-245.

24. Slamon D, Eiermann W, Robert N, et al. Adjuvant trastuzumab in HER2positive breast cancer. $N$ Engl J Med. 2011;365(14):1273-1283.

25. Canonici A, Gijsen M, Mullooly M, et al. Neratinib overcomes trastuzumab resistance in HER2 amplified breast cancer. Oncotarget. 2013;4(10):1592-1605.

26. Goldhirsch A, WoodWC, Coates AS, Gelber RD, Thórlimann B, Senn HJ. Strategies for subtypes - dealing with the diversity of breast cancer: highlights of the St Gallen international expert consensus on the primary therapy of early breast cancer 2011. Ann Oncol. 2011;22: 1736-1747.

27. Dawood S, Broglio K, Buzdar AU, Hortobagyi GN, Giordano SH. Prognosis of women with metastatic breast cancer by HER2 status and trastuzumab treatment: an institutional-based review. J Clin Oncol. 2010;28:92-98.

28. Roukos DH. Trastuzumab and beyond: sequencing cancer genomes and predicting molecular networks. Pharmacogenomics J. 2011;11(2): 81-92.

29. Gianni L, Dafni U, Gelber RD, et al. Treatment with trastuzumab for 1 year after adjuvant chemotherapy in patients with HER2-positive early breast cancer: a 4-year follow-up of a randomised controlled trial. Lancet Oncol. 2011;12:236-244.

30. Goldhirsch A, Gelber RD, Piccart-Gebhart MJ, et al. 2 years versus 1 year of adjuvant trastuzumab for HER2-positive breast cancer (HERA): an open-label, randomised controlled trial. Lancet. 2013;382(9897):1021-1028.
31. Cancer Research UK. A trial comparing 6 months and 12 months of trastuzumab (Herceptin) for early breast cancer (PERSEPHONE). http://www.cancerresearchuk.org/cancer-help/trials/a-trial-comparing6-months-and-12-months-of-trastuzumabfor-early-breast-cancer (accessed November 27, 2012).

32. Pivot X, Romieu G, Deblet M, et al. 6 months versus 12 months of adjuvant trastuzumab for patients with HER2-positive early breast cancer (PHARE): a randomized Phase 3 trial. Lancet Oncol. 2013;14(8):741-748.

33. Joensuu H. Duration of adjuvant trastuzumab: shorter beats longer. Lancet. 2013;382(9897):1010-1011.

34. Geyer CE, Forster J, Lindquist D, et al. Lapatinib plus capecitabine for HER2-positive advanced breast cancer. $N$ Engl J Med. 2006;355(26): 2733-2743.

35. Zheng Y, Zhang C, Croucher D, et al. Temporal regulation of EGF signalling networks by the scaffold protein Shc1. Nature. 2013;499(7457):166-171.

36. Sapra P, Betts A, Boni J. Preclinical and clinical pharmacokinetic/ pharmacodynamic considerations for antibody-drug conjugates. Expert Rev Clin Pharmacol. 2013;6(5):541-555.

37. Junttila TT, Li G, Parsons K, Phillips GL, Sliwkowski MX. Trastuzumab-DM1 (T-DM1) retains all the mechanisms of action of trastuzumab and efficiently inhibits growth of lapatinib insensitive breast cancer. Breast Cancer Res Treat. 2011;128:347-356.

38. Sassoon I, Blanc V. Antibody-drug conjugate (ADC) clinical pipeline: a review. Methods Mol Biol. 2013;1045:1-27.

39. Reichert JM. Antibodies to watch in 2013. Mid-year update. MAbs. 2013;5(4):513-517.

40. Mullard A. Maturing antibody-drug conjugate pipeline hits 30. Nat Rev Drug Discov. 2013;12:329-332.

41. Diéras V, Bachelot T. The success story of trastuzumab emtansine, a targeted therapy in HER2-positive breast cancer. Target Oncol. Epub July 14, 2013.

42. Burris HA 3rd, Rugo HS, Vukelja SJ, et al. Phase II study of the antibody drug conjugate trastuzumab-DM1 for the treatment of human epidermal growth factor receptor 2 (HER2)-positive breast cancer after prior HER2-directed therapy. J Clin Oncol. 2011;29:398-405.

43. Krop IE, Lo Russo P, Miller K, et al. A Phase II study of trastuzumabDM1 (T-DM1), a novel HER2 antibody-drug conjugate, in patients with HER 2+ metastatic breast cancer who were previously treated with an anthracycline, a taxane, capecitabine, lapatinib, and trastuzumab. Poster presented at: Annual San Antonio Breast Cancer Symposium. Cancer Res. 2009;70(Suppl):abstract 710.

44. Hurvitz SA, Dirix L, Kocsis J, et al. Phase II randomized study of trastuzumab emtansine versus trastuzumab plus docetaxel in patients with human epidermal growth factor receptor 2-positive metastatic breast cancer. J Clin Oncol. 2013;31(9):1157-1163.

45. Verma S, Miles D, Gianni L, et al. Trastuzumab emtansine for HER2-positive advanced breast cancer. N Engl J Med. 2012;367(19): 1783-1791.

46. Ellis PA, Barrios CH, Im Y, Patre M, Branle F, Perez EA. MARIANNE: a Phase III, randomized study of trastuzumab-DM1 (T-DM1) with or without pertuzumab $(\mathrm{P})$ compared with trastuzumab $(\mathrm{H})$ plus taxane for first-line treatment of HER2-positive, progressive, or recurrent locally advanced or metastatic breast cancer (MBC). J Clin Oncol. 2011; 29(Suppl 15):abstract TPS102.

47. Hoffmann-La Roche. A study of trastuzumab emtansine in comparison with treatment of physician's choice in patients with HER2-positive breast cancer who have received at least two prior regimens of HER2directed therapy (TH3RESA). Available from: http://clinicaltrials. gov/show/NCT01419197. NLM identifier: NCT01419197. Accessed January 27, 2014.

48. Hoffmann-La Roche. A study of trastuzumab emtansine versus trastuzumab as adjuvant therapy in patients with HER-2 positive breast cancer who have residual tumor in the breast of axillary lymph nodes following preoperative therapy (KATHERINE). Available from: http:// clinicaltrials.gov/show/NCT01772472. NLM identifier: NCT01772472. Accessed January 27, 2014. 
49. Baselga J, Gelmon KA, Verma S, et al. Phase II trial of pertuzumab and trastuzumab in patients with human epidermal growth factor receptor 2-positive metastatic breast cancer that progressed during prior trastuzumab therapy. J Clin Oncol. 2010;28:1138-1144.

50. Baselga J, Cortés J, Kim SB, et al. Pertuzumab plus trastuzumab plus docetaxel for metastatic breast cancer. N Engl J Med. 2012;366: 109-119.

51. Tebbutt N, Pedersen MW, Johns TG. Targeting the ERBB family in cancer: couples therapy. Nat Rev Cancer. 2013;13(9):663-673.

52. Blackwell KL, Burstein HJ, Storniolo AM, et al. Randomized study of Lapatinib alone or in combination with trastuzumab in women with ErbB2-positive, trastuzumab-refractory metastatic breast cancer. J Clin Oncol. 2010;28:1124-1130.

53. Baselga J, Bradbury I, Eidtmann H, et al. Lapatinib with trastuzumab for HER2-positive early breast cancer (NeoALTTO): a randomised, open-label, multicentre, phase 3 trial. Lancet. 2012;379:633-640.

54. $\mathrm{Ku} \mathrm{CS}$, Roukos DH. From next-generation sequencing to nanopore sequencing technology: paving the way to personalized genomic medicine. Expert Rev Med Devices. 2013;10(1):1-6.

55. Heinz S, Romanoski CE, Benner C, et al. Effect of natural genetic variation on enhancer selection and function. Nature. 2013; 503(7477):487-492.

56. Hindorff LA, Sethupathy P, Junkins HA, et al. Potential etiologic and functional implications of genome-wide association loci for human diseases and traits. Proc Natl Acad Sci U S A. 2009;106:9362-9367.

57. Roukos DH, Baltogiannis GG, Baltogiannis G. Mapping inherited and somatic variation in regulatory DNA: new roadmap for common disease clinical discoveries. Expert Rev Mol Diagn. 2013;13(6):519-522.

58. Maurano MT, Humbert R, Rynes E, et al. Systematic localization of common disease-associated variation in regulatory DNA. Science. 2012; 337(6099):1190-1195.
59. Roukos DH. Cancer heterogeneity and signaling network-based drug target. Pharmacogenomics. 2013;14(11):1243-1246.

60. Gerstein MB, Kundaje A, Hariharan M, et al. Architecture of the human regulatory network derived from ENCODE data. Nature. 2012;489(7414):91-100.

61. Neph S, Stergachis AB, Reynolds A, Sandstrom R, Borenstein E, Stamatoyannopoulos JA. Circuitry and dynamics of human transcription factor regulatory networks. Cell. 2012;150(6):1274-1286.

62. ENCODE Project Consortium, Bernstein BE, Birney E, Dunham I, Green ED, Gunter C, Snyder M. An integrated encyclopedia of DNA elements in the human genome. Nature. 2012;489(7414):57-74.

63. Sanyal A, Lajoie B, Jain G, Dekker J. The long-range interaction landscape of gene promoters. Nature. 2012;489(7414):109-113.

64. Yosef N, Shalek AK, Gaublomme JT, et al. Dynamic regulatory network controlling TH17 cell differentiation. Nature. 2013; 496(7446):461-468.

65. Cheng TM, Gulati S, Agius R, Bates PA. Understanding cancer mechanisms through network dynamics. Brief Funct Genomics. 2012; 11(6):543-560.

66. Ball P. DNA: celebrate the unknowns. Nature. 2013;496(7446): 419-420.

67. Roukos DH. Genome network medicine: new diagnostics and predictive tools. Expert Rev Mol Diagn. 2013;13(7):643-646.

68. The Cancer Genome Atlas Research Network. Comprehensive molecular characterization of human colon and rectal cancer. Nature. 2012;487:330-337.

69. International Cancer Genome Consortium, Hudson TJ, Anderson W, Artez A, et al. International network of cancer genome projects. Nature. 2010;464(7291):993-998.
OncoTargets and Therapy

\section{Publish your work in this journal}

OncoTargets and Therapy is an international, peer-reviewed, open access journal focusing on the pathological basis of all cancers, potential targets for therapy and treatment protocols employed to improve the management of cancer patients. The journal also focuses on the impact of management programs and new therapeutic agents and protocols on

\section{Dovepress}

patient perspectives such as quality of life, adherence and satisfaction The manuscript management system is completely online and includes a very quick and fair peer-review system, which is all easy to use. Visit http://www.dovepress.com/testimonials.php to read real quotes from published authors. 